\title{
MORPHOLOGY AND MICROCIRCULATORY STRUCTURE OF THE URETHRA OF THE PIG: Urologic surgical interest
}

Fréjus Séry ${ }^{1}$, Henry Sick ${ }^{2}$

1- Laboratory of anatomy, Bouaké Alassane OUATTARA University, Graduate school of Medicine Bouake Côte d'Ivoire

2- Laboratory of Normal Anatomy, Strasbourg Medical School, France

Correspondence to Frejus Séry: 01BPV18 Bouaké 01/ Côte d'Ivoire. frejussery05@gmail.com, Phone: 0022507839159.

\begin{abstract}
The urethra of the sow can play an important role in the surgical revascularization or in the reconstructive surgery. However, the urethra can be easily injured if it possessed well architecture and vascularization. We investigated the morphological structure and vasculature features of the urethra to improve surgical results. Eight pelvises of sow were investigated. The block was cuts according to the segments of the urethra. They were made transparent according to the sagittal and transvers planes and examined trough Chinese ink agar injection. The adventia of both portions of the urethra of the sow (pelvic and perineal) were dependent on the collaterals of the internal iliac arteries and internal pudendal arteries. The veins of the periphery of the urethra derived from the muscle layers and the chorion and ran into a network in contact with arteries. The arteries of the striated muscle layer originated from the adventitial arteries of the anterior and lateral regions of the urethra. The chorion was depended on long vessels from the adventitious network and the terminal branches of the vessels of the inner longitudinal muscle layer. The arteries of the urethrovaginal septum were thin and came from the branches of the vaginal artery. The urethra of the sow was sheathed by the pelvic fascia lined with the elevated muscle of anus. They were well vascularized and can be safety exposed and preserved during experimental surgical dissection with careful drilling.
\end{abstract}

Keys words: Sow urethra, morphology, structure, microvascularization.

\section{INTRODUCTION}

Pigs are non-human vertebrates useful for anatomy and physiology experiments because of their similarities with humans. Similarly, many surgical techniques, kidney and liver transplantation have been developed on pigs before being performed on humans. The valves used in the replacement of the surgical heart valves were pig valves. Pig skin was often used as a temporary skin graft for serious burns. Following the anatomical work performed on the urethra of the sow by some authors and clinical, urodynamic,

\section{MATERIAL AND METHODS}

The sow was chosen as a study model in its Menschen and White variety. Eight pelvises of sow of both varieties were injected with Chinese ink agar from the internal iliac artery. Then placed in freezing to allow the fixation of the injected product. 72 hours later, the neurophysiological and pharmacological researches, (Barone, 1996; Bridgewater et al, 1993; Jacek et al, 1991 ; Nickel, 1981; Graziotty et al 2007; Nitsche, et al 1971; Zini et al 2006; Whitmore et al, 1984). The present study focuses on the whole of the two portions of the urethra both structural and microcirculatory. That study was performed on a cadaver of the sow donated to our laboratory for students' dissection and research.

vesico-urethral block was extensively removed and then fixed with $20 \%$ formalin. The block was cut according to the segments (proximal, middle and distal) of the urethra. On each segment were made thick sections of 12 microns according to the transverse and 
sagittal planes. They were made transparent according to Spalteholz method, then stained with haemalun-eosin (4 cases) or with Masson's trichrome (4 cases). All sections were examined with a stereoscopic magnifying glass and photographed using the Zeiss photomicroscope.

\section{RESULTS}

The urethra followed the bladder and was located in front of the vagina and uterus. It was sheathed by the pelvic fascia lined with the elevating muscle of the anus. It had two portions, pelvic and perineal. The muscularis was an extension of the plexiform muscular layer of the bladder. The urethra in both varieties had a structure with three tunics (adventia, muscularis and mucosa) (figure 1). Laterally, the adventis was reinforced outside by the pelvic fascia through which the urethra comes into contact with the pelvic organs. The adventis, muscular and mucous Tunics of the urethra of the pig are characterized by their histological and microcirculatory appearance. (figure 2).

The adventis of both portions of the urethra of the sow (pelvic and perineal) were dependent on the collaterals of the internal iliac arteries and internal pudendal arteries. The pedicles of the pelvic part of the urethra originated from the branches of the urethral, vesical, and vaginal arteries. The pedicles of

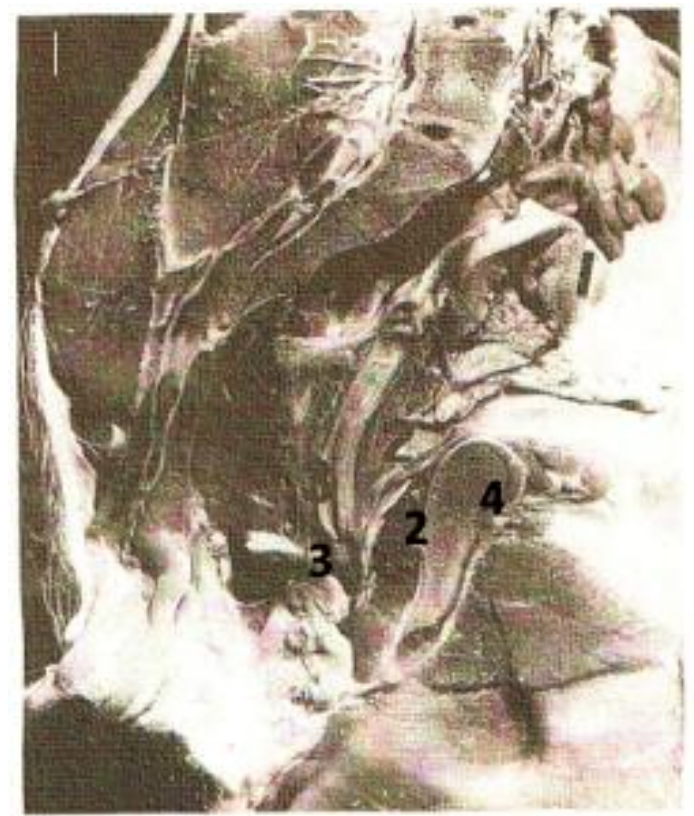

Figure 1: Sagittal section of the pelvic of the sow. at three months. Bladder; 2- Urethral wall;3- Vagina; 4Pelvic fascia the perineal part depended on the arteries of the vestibular bulb and the deep dorsal artery of the clitoris. The collateral and terminal branches of the pelvic and perineal portions of the arteries gave ascending and descending branches along the anterior and lateral surfaces of the urethra. These branches exchanged anastomoses between them and with those on the opposite side. Thus, was established on the whole circumference of the urethra, an anastomotic system between these two portions. This system consisted of arterial mesh networks whose morphology differed according to the regions: proximal, middle and distal of the urethra. The veins of the periphery of the urethra derived from the muscle layers and the chorion and ran into a network in contact with arteries. The clean adventitious venous network consisted of veins of small calibers arranged in transversely oriented meshes that ended in large veins that joined the veins of the preurethral and latero-vaginal plexuses (figure 3).

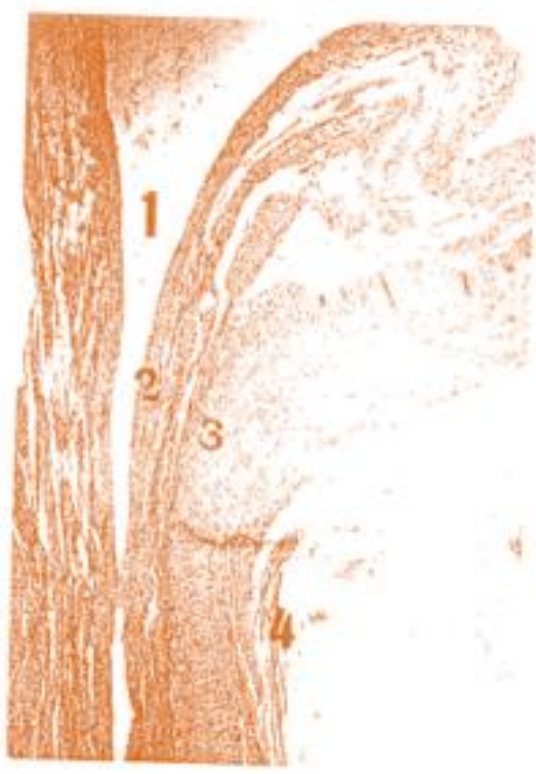

Figure 2: Histologic sagittal section of the Urethra of the Sow. at three months, Hemalun-eosine (X4). 1-Vesico urethra luminen; 2- Mucous tunic; 3Muscularis tunic ; 4-Adventis tunic 


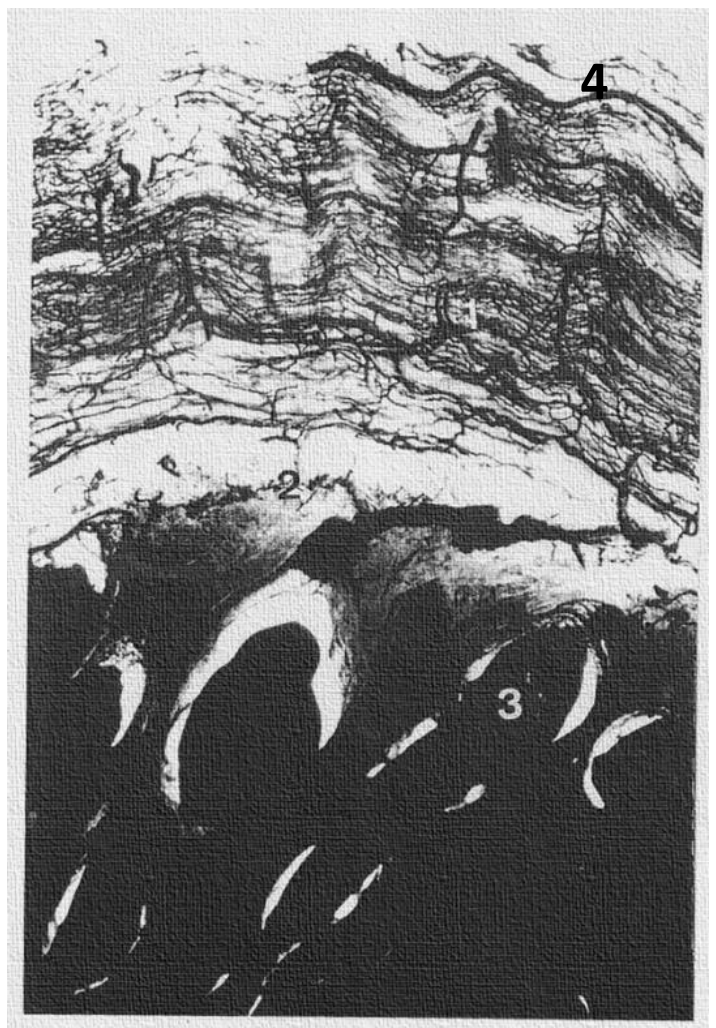

Figure 3: Transverse section of the urethra of the sow at three months. Injection-Transparification(X4). 1-Muscular circular vessel; 2-Longitudinal vessel; 3-Longitudinal vessel of the submucosa ; 4-Adventitious vein.

Some large caliber veins, such as those of the chorion or the longitudinal layer, ran directly into the peripheral veins (figure 4). The arteries of the striated muscle layer originated from the adventitial arteries of the anterior and lateral regions of the urethra. Those of the outer striated and smooth muscle layer were transverse, parallel to the muscle fibers and anastomosed between them via the inter- fascicular and fascicular arteries and with the arteries of the longitudinal muscle fibers (figure 3). Some arteries were destined primarily for the longitudinal muscle layer. They crossed the intermuscular zone or ended there in two or three branches. During their course, they abandoned inter-fascicular collateral branches destined for the smooth longitudinal layer.

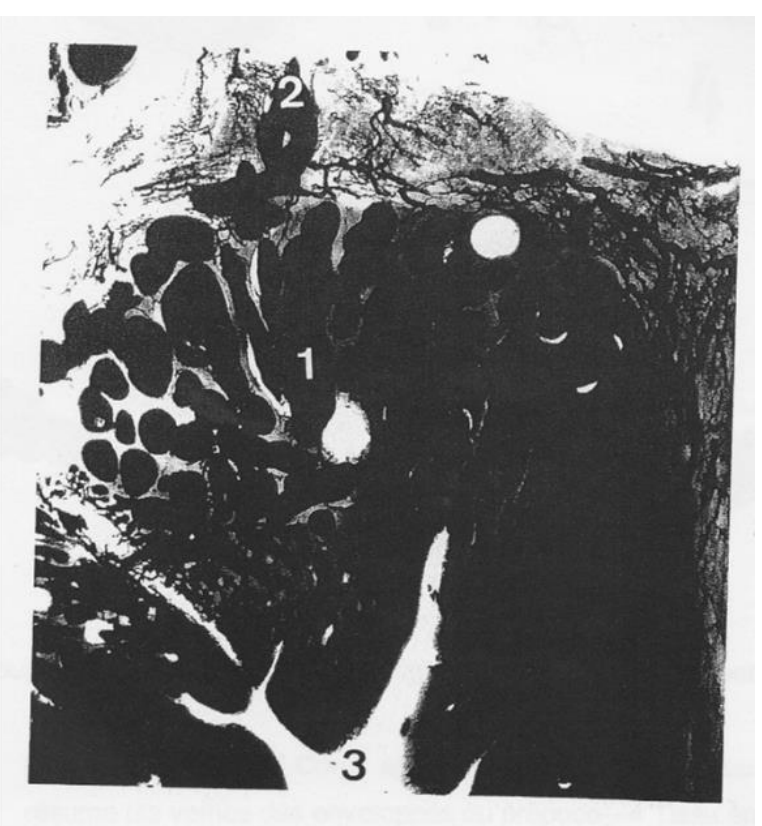

Figure 4: Vascularization of the wall of the lower third of the urethra of the sow. Cross section (X10) 1-Chorion vein; 2- Muscle vein; 3-Urethral wall 
They thus participated in the constitution of an intermuscular arterial network. This network was dependent on the long vessels from the adventitial network and terminal branches of the vessels of the internal longitudinal muscle layer (figure 5). Other longer arteries crossed the striated and smooth muscle layers to reach the chorion directly. During their course, they also abandoned intermuscular arteries. The arteries of the plexiform muscle layer of the bladder were prolonged with those of the longitudinal layer of the urethra. The muscle veins were more numerous and followed the arteries of larger caliber. They were more richly anastomosed than these. The venous network took a disposition comparable to that described in the arteries. These veins consisted of the confluence of the fascicular, inter-fascicular and chorionic veins. The chorion was dependent on long vessels from the adventitious network and the terminal branches of the vessels of the inner longitudinal muscle layer. The arteries of the chorion and the mucosa came from the arteries of the longitudinal muscle layer and the adventitial arteries of the urethra. Arranged according to the axis of the urethra in the chorion, they were parallel to each other, little anastomosed, and gave two to three collaterals to each urethral tunic.

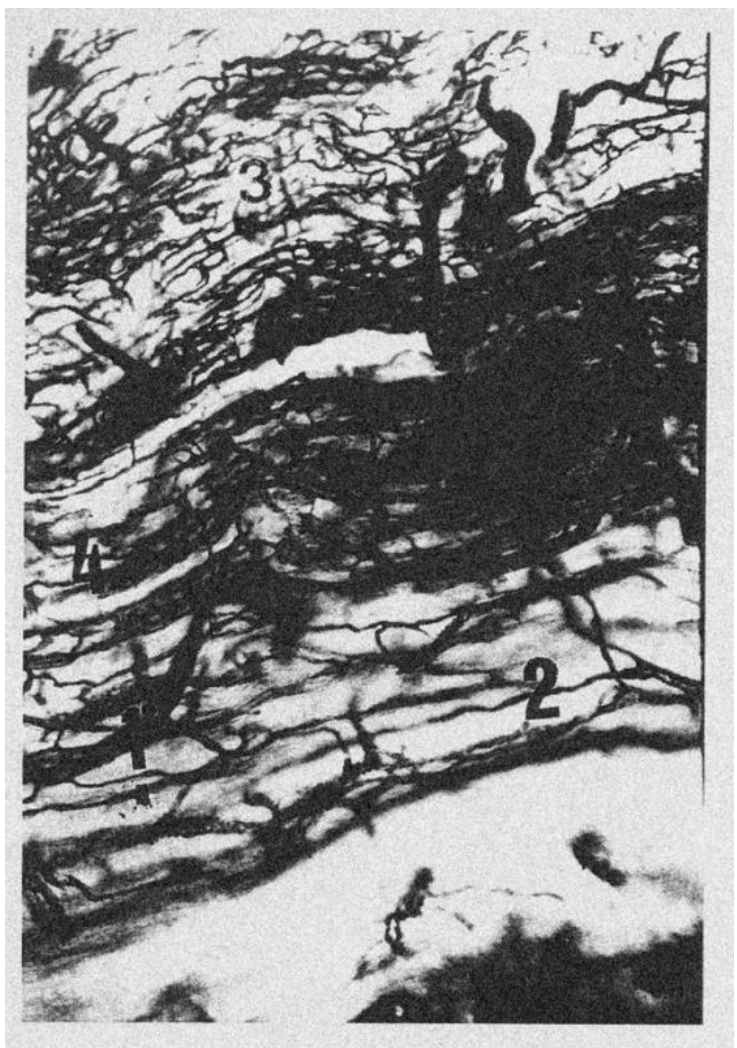

The veins of the chorion followed the arteries. They were particularly dilated and their caliber was two to three times that of the arteries. They occupied the entire chorion whose thickness doubled in the middle third of the urethra. The venous drainage of each urethral fold consisted of two or three large veins that ran directly into the longitudinal veins of the chorion. The density and caliber of the venous system of the chorion grew as the middle and perineal part of the urethra was approached (figure 3-5). The arteries of fine caliber ran into a capillary network with regular meshes that married the reliefs of the mucosa. The veins of the mucosa were of a caliber a little larger than that of the arteries they followed. They were little anastomosed together and joined the veins of the chorion. In addition, they followed the arteries and then drained to the periphery of the urethra and even directly to the lateral plexuses of the vagina. The arteries of the urethrovaginal septum were thin and came from the branches of the vaginal artery. They were longitudinal and anastomosed little with the branches of the muscular arteries of the anterior wall of the vagina. The vascular poverty of the urethrovaginal septum correlated with its urethrovaginal areolar structure (figure 3,4 ).
Figure 5: Muscular vessel of the tunic at three months. Injection-Transparification. 1-Gross muscular vein; 2-Fine muscular vessel ; 3-Fascicular muscular vessel ; 4- Inter fascicular muscular vessel 


\section{DISCUSSION}

The discussion is anatomical and clinical. Anatomically: The walls of the urethra of the sow were studied by (Barone, 1996; Dasset al. 2001; Zini et al. 2006; Nickel, 1981). In these authors, the striated external sphincter of the urethra of the sow extended from the bladder neck to the central tendon of the perineum. It surrounded the urethral lumen and was intimately associated with the urethral muscularis. It is thick on the ventral surface of the urethra more than on its dorsal surface (Strasser et al. 2000). It is related to the pelvic sagittal blade (constantinescou, 2004; Bridgewater et al, 1993; Neuhaus et al, 2001). This case was similar to the case reported by (Strasser et al 2000).

At the vascular level, the urethra of the sow is vascularized by the lower bladder artery (Nickel et al. 1981; Constantinescou et al, 2004). In the dog and some domestic animals, this artery originated from the urogenital or vesico-prostatic artery in the human, branch of the internal iliac artery (Roberts et al, 1967; Trappe, 1984; Neuhaus et al, 2001). In our observations there was no vascularization from the middle bladder as described by (Dasset et al,2001). Our observations were identical to those of (Zini et al, 2006; Strasser et al, 2000; Wang et al, 1999; Hossler et al 1995) where the lower bladder artery vascularized the urethra of the sow. similarly, it has been reported that in carnivores this lower vesical artery irrigates the prostate and the pelvic urethra. The urethra of the sow was vascularized by collateral and terminal branches of the arteries of the plexiform layer of the bladder, arteries of sagittal vascular blades and clean arteries of the periphery of the urethra. The arterial ramifications were anastomosed and gave rise to a network of dense and regular meshes extended over the whole height of the urethra and inside the tunics of the wall.

The venous vasculature had a deep, superficial venous system and formed a centripetal current, which ended in one or two large adventitial veins that ran into the preurethral and latero-vaginal plexus, as in women. The urethrovaginal septum is equally poor in vessels. There was a contrast between the circulation of the chorion and that of the urethral muscularis. It was a weakly anastomotic vascular network. Clinically: During the last decades, thanks to experimental studies on sows, enormous progress in the sow has been achieved (Jacek et al, 1991; Bridgewater et al, 1993; Zini et al, 2006). Neurological, anatomical and histological studies of the pelvic urethra of the sow have established inclusion criteria for maintaining urinary continence in the proximal two-thirds of the urethra. Despite these criteria and the dissection techniques performed in women and other non-human vertebrates (Barone, 1996; Kirstic, 1991; Boriakchanyavat et al, 1997; Gosling et al, 1981; Elbadawi et al, 1996; Dasset et al, 2001; Whitmore et al, 1984) other neurological, anatomical and urodynamic studies have been done in domestic mammals (dogs and sows) (Bridgewater et al, 1993; Constantinescou, 2004; Nickel, 1981; Nitsche, 1971) which revealed that, in addition to sphincteric, muscle and nervous factors, vascular factors are involved in the urinary continence of the sow. We did not carry out a parallel clinical study, but from our findings some points of interest came out. The urethra of the sow is a well vascularized organ and the vessels involved in its irrigation constitute a remarkable functional unit without any solution of continuity. The increase in venous density of the chorion along the urethra could probably explain the urinary continence of this non-human vertebrate. Medically for the moment, no vasodilator treatment is yet undertaken in the woman's urethra. This could be carried out experimentally in the sow from the microvasculature data of its urethra.

In conclusion, the morphological, structural and microcirculatory study of the urethra of the sow raises many points of interest in urological surgery. Research in the field of urinary continence has been performed on histological and histochemical techniques. Current data on the microvasculature of the 
urethra of the sow make it possible to compare the urethral structure of the sow and that of the woman. Clinical investigations could provide a guarantee for this study and improve the replacement surgery of the urethra. This maybe a reference for interpreting the microvascular connection of the urethra of the sow in relation to neighboring organs, as well as for other morphological studies on non-human vertebrates.

Acknowledgements: The authors are grateful to the personality of the breeding center donated the sow without any economic benefit to Bouake University graduate School of Medicine.

Conflicts of interest: The authors declare that they have no conflicts of interest.

\section{REFERENCES}

1. Barone R. 1996. Anatomie Comparée des Mammifères Domestiques, Angiologie Vigot. Paris; pp 357-385.

2. Boriakchanyavat S, Baskin LS, Kogan BA. 1997. Smooth and striated muscle development in the intrinsic urethral sphincter. J Urol, 158: 1119-1122.

3. Bridgewater M, Macnerl HD, Brading AF.1993. Regulation of tone in pig urethral smooth muscle. J Urol, 150: 223-28.

4. Constantinesco GM. 2004. veterinary Anatomy of Domestic Mammals, Textbook and Color Arlas. AZ Druck and Valerg. Germany; pp 377-379.

5. Dasset N, McMurray G, Greenland JE. 2001. Morphological aspects of the female pig bladder neck and urethra: quantitative analysis using computer assisted 3-dimensional reconstructions. J Urol, 165: 1294-1299.

6. Elbadawi A. 1996. Funcional anatomy of the organ of micturition. Urol Clin North am, 23: 177-210.

7. Gosling JA, Dixon JS, Critchley HO. 1981. A comparative study of the human external sphincter and periurethra llevatori muscles. $\mathrm{Br}$ J Urol, 53: 35-41.

8. Graziotti GH, Menendez JMR, Rios CM, Victoria CL. 2007. Distribution of the internal pudendal artery in male and female llama (Lama glama). Annals of Anatomy, 139: 295-298.

9. Hossler FE, Monson FC. 1995. Microvasculature of the rabbit urinary bladder. Anatomical Record, 243: 438-448.

10. Jacek L. mostwin JL, Karim OMA, Koeveringe VG. Brook EL 1991. The guinea pig as a model of gradual urethtal obstruction. J Urol, 145: 845-858.

11. KIRSTIC R. V. 1991.Human microscopic anatomy. Atlas, for students of medicine and biology. Springer Verlag; p 585.

12. Neuhaus J, Dorschner W, Mondry J. 2001. Comparative of the male guinea-pig and human lower urinary tract: histomorphplogy and three- dimensional reconstruction. Anat Histol Embryol, 30: 185-192.

13. Nickel R, Schummer A, Seiferle E. 1981. The anatomy of the Domestic Animals. Vol. 3. Verlag Paul Parey. Berlin-Hamburg, 176-181.

14. Nitsche T, Preuss F. 1971. Main branches of the internal iliac artery in man and domestic mammals in a comparative anatomical study. Anatomischer Anzeiger, 128: 439-453.

15. Roberts WH, Gene LK. 1967. Comparative Study of human internal iliac Artery based on adachi classification. Anat Rec, 158: 191-196.

16. Strasser H, Ninkovic M, Hess M. 2000. Anatomic and functional studies of the male and female urethral sphincter. World J Urol, 18: 324-329. 
17. Trappe K. 1984. Origin and course of the perineal blood vessels in swine. Tieraztliche Hochschule. Hannover; pp 77.

18. Wang B, Bhadra N, Grill WM. 1999. Functional anatomy of the mal feline urethra: morphological and physiological correlations. J Urol, 161: 654-659.

19. Whitmore I, Gosling JA. 1984. A comparison between the physiological and histochemical characterization of urethral striated muscle in the guinea pig. Pflugers Arch, 400: 40-43.

20. Zini L, Lecoeur C, Swieb S. 2006. The striated urethral sphincter of the pi shows morphological and functional characteristics essential for the evaluation of treatments for sphincter insufficiency. J Urol, 176: 2729- 2735. 